Mittheilung ans dem chemischen Institut der Dniversität Bonn.

\title{
Ueber Eigenschaften,
} insbesondere das Drehungsvermögen des Saccharins und der Alkalisaccharinate; von Eberhard Rimbach und Edmund Heiten.

(Eingelaufen am 19. Januar 1908.)

Zwecks einer anderweitigen Untersuchung hatten wir das Péligot'sche ${ }^{1}$ ) Saccharin $\mathrm{C}_{6} \mathrm{H}_{10} \mathrm{O}_{5}$, das Lacton der Saccharinsăure, in grösseren Mengen dargestellt. Ueber die physikalischen Eigenschaften, insbesondere die Drehung dieses optisch activen Körpers finden sich nun in der Literatur nur ganz vereinzelte Angaben. Wir benutzten daher diese Gelegenheit zu einer eingehenden Messung der Drehung des Saccharins and einiger Alkalisaccharinate, in ihrer Abhängigkeit von Art und Menge des Lösungsmittels, Lichtart und Temperatur; weiter wurde die elektrische Leitfăhigkeit wässriger Lösungen dieser Substanzen bestimmt, endlich konnten noch ihre krystallographischen Constanten festgelegt werden.

\section{Darstellung des Saccharins.}

Das Verfahren von Péligot bezw. Scheibler ${ }^{2}$ ) hochen einer Lösung von Lävulose oder invertirtem Rohrzucker

') Péligot, Compt. rend. 89, 920; 00, 1141 (188ט).

2) Scheibler, Ber. d. dentsch. chem. Ges. 18, 2212 (1880). 
mit Aetzkalk - ergab uns trotz grösster Vorsicht selbst bei Anwendung reiner Lävulose, nur sehr unbefriedigende Resultate. Besser gelang die Umwandlung des Invertzuckers in Saccharin nach der von Kiliani ${ }^{3}$ ) herrührenden Vorschrift, durch monatelanges Stehenlassen der mit Kalkhydrat versetzten Flussigkeit bei gewöhnlicher Temperatur. Unsere Ausbeute erreichte allerdings nicht die von Kiliani; je $5 \mathrm{~kg}$ Rohrzucker lieferten uns in mehreren Versuchen höchstens je $100 \mathrm{~g}$ reines Saccharin in Form grosser, sehr gut ausgebildeter, farbloser Krystalle. Deren Schmelzpunkt lag bei $160-161^{\circ}$, genau entsprechend den Angaben von Péligot und Scheibler (a. a. 0.). Ihre Krystallform festzustellen batte Herr Privatdocent Dr. A. Fock in Berlin die Freundlichkeit, wofür ihm auch an dieser Stelle bestens gedankt sei.

$$
\begin{gathered}
\text { Saccharin. } \\
\text { "Krystallsystem : Rhombisch. } \\
\mathrm{a}: \mathrm{b}: \mathrm{c}=0,9294: 1: 1,3531 . \\
\text { Beobachtete Formen : } \\
\mathrm{q}=(011), \mathrm{r}=(101), 1=(102) \text { und } \mathrm{m}=(110) .
\end{gathered}
$$

Die Krystalle sind prismatisch nach der Axe a und bis zu $10 \mathrm{~mm}$ lang und $4 \mathrm{~mm}$ dick. Von den angegebenen Formen herrschen $\mathrm{q}(011)$ und $\mathrm{r}(101)$ regelmässig vor, während 1(102) zurucktritt oder ganz fehlt; $m(110)$ wurde nur an einzelnen Individuen in minimaler Ausdehnung beobachtet.

$$
\begin{array}{rcc} 
& \text { Beobachtet: } & \text { Berechnet: } \\
\mathrm{q}: \mathrm{q}=(011):(01 \overline{1})=72^{\circ} 56^{\prime} & - \\
\mathrm{r}: \mathrm{r}=(101):(10 \overline{1})=68^{\circ} 58^{\prime} & - \\
\mathrm{l}: \mathrm{r}=(102):(101)=19^{\circ} 23^{\prime} & 19^{\circ} 27^{\prime} \\
\mathrm{q}: \mathrm{r}=(011):(101)=70^{\circ} 24^{\prime} & 70^{\circ} 20^{\prime} \\
\mathrm{q}: 1=(011):(102)=62^{\circ} 3^{\prime} & 62^{\circ} 2^{\prime} \\
\mathrm{m}: \mathrm{q}=(110):(011)=52^{\circ} 28^{\prime} & 52^{\circ} 51^{\prime} \\
\mathrm{m}: \mathrm{m}=(110):(1 \mathrm{I} 0)=94^{1} / \mathrm{q}^{\circ} \mathrm{ca} . & . . & 94^{\circ} 12^{\prime}
\end{array}
$$

Spaltbarkeit deutlich nach $\mathrm{r}(101)$.

3) Kiliani, Ber. d. deutsch. chem. Ges. 15, 2954 (1882). 
Ebene der optischen Axen für blau $=(010)$, für roth $=$ (100), für gelbes Natriumlicht ist die Substanz so gut wie cinaxig.

$2 \mathrm{E}$ für blau und roth $=$ circa $2-3^{0 \text { 's. }}$

\section{Versuchsanordnung.}

Für die nachstehend beschriebenen Messungen sei Folgendes bemerkt. $\mathrm{Zu}$ den Drehungsbestimmungen diente ein Lippich'scher Halbschattenapparat mit dreitheiligem Gesichtsfelde mit Ablesung auf $1 / 100$ Grad. Als Lichtquelle benutzten wir entweder Natriumlicht, das durch eine Schicht von Kaliumbichromat gereinigt wurde, oder zur Herstellung von Lichtarten anderer Wellenlänge eine Auer'sche Gasglühlampe mit vorgelegten Landolt'schen Strahlenfiltern ${ }^{4}$ ). (Optischer Schwerpunkt für roth: $\lambda=665,5$; grün: $\lambda=533,0$; hellblau : $\lambda=488,5$; dunkelblau: $\lambda=448,2$.) Die Ablenkung wurde bestimmt in Wasserbadröhren; da die für den Ablenkungswinkel $\alpha$ angeführten Werthe das Mittel aus mindestens zehn Ablesungen des Nullpunktes und der Ableukung sind, so kann für Natriumlicht der wahrscheinliche Fehler von $\alpha$ auf $\pm 0,01^{\circ}$ gesetzt werden. Bei den anderen Messungen wird durch die nicht vollständige Homogenität und durchschnittlich etwas geringere Intensität des angewendeten Lichtes die Unsicherheit, besonders für dunkelblau, etwas grösser. Die Herstellung der Lösungen erfolgte entweder direct nach Concentrationen im Messkölbchen oder nach Gewichtsprocentgehalt unter gleichzeitiger Ermittelung der Dichte der Lösung mittelst eines circa $14 \mathrm{ccm}$ fassenden Sprengel'schen Pykuometers.

Die elektrischen Leitfähigkeitsbestimmungen sind in üblicher Weise mittelst der Wheatstone'schen Brücke nach Kohlrausch's Wechselstrommethode ausgefuhrt. Das Widerstandsgefäss befand sich in einem 0 stwald'schen Thermostaten von genau $25^{\circ}$. Die angegebenen specifischen Leitfähig-

4) La nd olt, Ber. d. deutsch. chem. Ges. 27, 2872 (1894). 
320 Rimbach u. Heiten, Eigenschaften, Drehungsvermögen

keiten bedeuten reciproke Ohms nach Abzug der Eigenleitfăhigkeit des Lösuugswassers.

Die Ergebnisse der Messungen, zunächst von wässriger Saccharinjosung, finden sich in nachfolgenden Tafeln.

Tafel I.

Saccharin in Wasser.

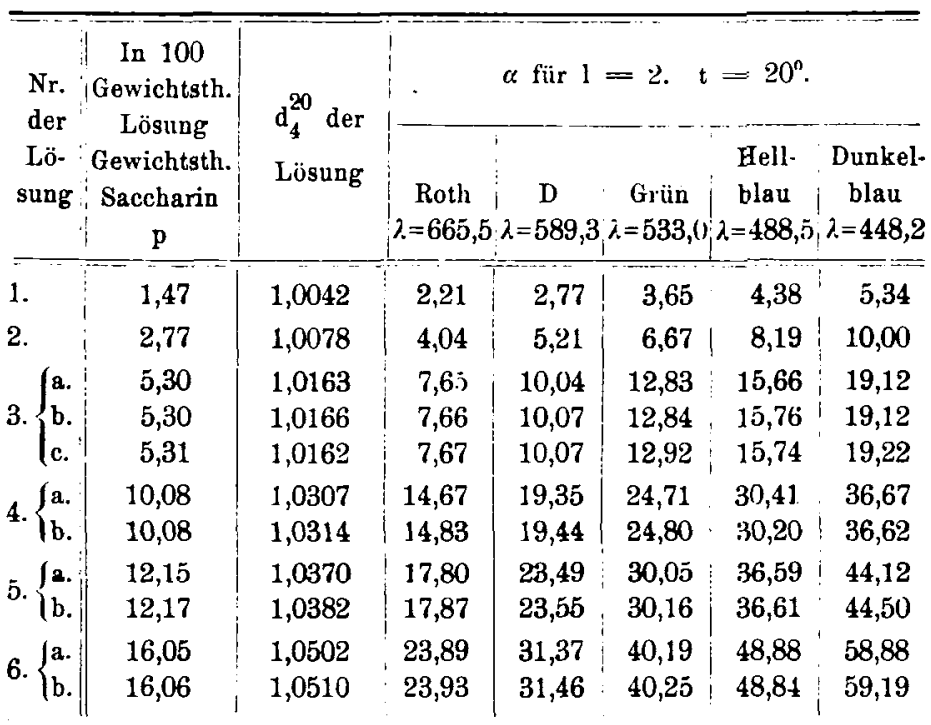

Für einige Concentrationen wurden die Bestimmungen $b$ bezw. c der Sicherheit wegen mit gesondert hergestellten Lösungen wiederholt. Benutzt man, was bei der naben Uebereinstimmung zulässig ist, die Mittel für $\mathrm{p}$ und $\alpha$, so erhält man schliesslich nachstehende Werthe für die specifischen Drehungen der wässrigen Saccharinlösung: 


\begin{tabular}{|c|c|c|c|c|c|c|c|}
\hline Nr. & \multirow[b]{2}{*}{$\mathbf{p}$} & \multicolumn{6}{|c|}{$[\alpha] 20^{\circ}$} \\
\hline $\begin{array}{c}\text { der } \\
\text { Lö- } \\
\text { sung }\end{array}$ & & Roth & D & Grün & Hellblau & $\begin{array}{c}\text { Dunkel- } \\
\text { blau }\end{array}$ & $\begin{array}{c}\text { Dispersions- } \\
\text { coëfflcient } \\
\text { Dunkelblau- } \\
\text { Roth }\end{array}$ \\
\hline 1. & 1,47 & 74,86 & 93,82 & 123,63 & 148,36 & 180,87 & 2,42 \\
\hline 2. & 2,77 & 72,36 & 93,31 & 119,40 & ]46,68 & 179,10 & 2,47 \\
\hline 3. & 5,303 & 71,05 & 93,32 & 119,32 & 145,82 & 177,68 & 2,50 \\
\hline 4. & 10,08 & 70,95 & 93,24 & 119,16 & 145,80 & 176,30 & 2,48 \\
\hline 5. & 12,16 & 70,68 & 93,21 & 119,30 & 145,04 & 175,60 & 2,48 \\
\hline 6. & 16,055 & 70,89 & 93,13 & 119,22 & 144,84 & 175,00 & 2,47 \\
\hline
\end{tabular}

Wie aus diesen Zahlen hervorgeht, stimmen die frther von Péligot ${ }^{5}$ ), Scheibler ${ }^{6}$ ) und Tollens ${ }^{7}$ ) an vereinzelten Lösungen gefundenen Drehungswerthe mit den betreffenden, von uns ermittelten Werthen gut uberein. Péligot fand $[\alpha]_{D}=+93,1^{\circ}$, Scheibler $[\alpha]_{D}=+93,8^{\circ}$ für 12-procentige Lösung, Tollens $[\alpha]_{D}=+93,1^{\circ}$ für $\mathrm{c}=4$. Mit steigender Concentration nimmt die specifische Drehung $a b$, jedoch nur in geringem Grade, besonders schwach bei Natrium:licht. Die Abnahme ist hier ungefähr von der Grössenordnung der Aenderung der Drehung mit der Concentration, die man beim Rohrzucker beobachtet. Verwendet man andere Lichtarten, so werden die Werthe der Drehungsänderung etwas höher. Die Rotationsdispersion bietet keine Anomalien; sie ist ein wenig grösser, als sie der Quarz für die gleichen Wellenlängen überall aufweist.

Im Anschlusse an diese Messungen bestimmten wir weiter die Drehung des Saccharins in verschiedenen anderen Lösungsmitteln. In den folgenden Tafeln sind die gefundenen Zahlen tbersichtlich zusammengestellt.

5) Péligot, Compt. rend. 90, 1141.

) Scheiblor, Ber. d. deutsch. chem. Ges. 13, 2216.

7) Tollens, Ber. d. deutsch. chem. Ges. 18, 1333. 
Tafel II.

Saccharin in Methylalkohol.

\begin{tabular}{|c|c|c|c|c|c|c|c|c|}
\hline \multirow{2}{*}{\multicolumn{3}{|c|}{\begin{tabular}{cc|} 
& In 100 \\
Nr. & Gewichtsth. \\
der & Lösung \\
Lö- & Gewichtsth. \\
sung & Sacchario \\
& $p$ \\
\end{tabular}}} & \multirow[t]{2}{*}{$\begin{array}{l}\mathrm{d}_{4}^{20} \mathrm{der} \\
\text { Lösung }\end{array}$} & \multicolumn{5}{|c|}{$\alpha$ für $I=2 . \quad t=20^{n}$} \\
\hline & & & & & $=589,3 \lambda=$ & & & \\
\hline $\begin{array}{l:}1 . \\
2 . \\
\text { 3. } \\
\text { 4. } \\
5 .\end{array}$ & $\begin{array}{r}3,05 \\
4,84 \\
6,37 \\
8,19 \\
11,05\end{array}$ & : & $\begin{array}{l}0,80 \dot{\mathbf{b}} 7 \\
0,8114 \\
0,8186 \\
0,8267 \\
0,8384\end{array}$ & $\begin{array}{r}3,68 \\
5,85 \\
7,71 \\
9,96 \\
13,60\end{array}$ & $\begin{array}{r}4,83 \\
7,63 \\
10,12 \\
13,07 \\
17,87\end{array}$ & $\begin{array}{l}, 18 \\
, 81 \\
2,98 \\
, 75 \\
, 84\end{array}$ & $\begin{array}{r}7, \\
11, \\
15\end{array}$ & \begin{tabular}{l|r}
30 & 9,24 \\
56 & 14,41 \\
21 & 19,11 \\
68 & 24,79 \\
88 & 33,91
\end{tabular} \\
\hline & & & & & {$[\alpha] 20^{\circ}$} & & & \\
\hline $\begin{array}{l}\text { ler } \\
\text { Gö- } \\
\text { ung }\end{array}$ & p & Roth & $\mathrm{D}$ & Giriün & Hellblau & $\begin{array}{c}\text { Dun } \\
\text { bla }\end{array}$ & & $\begin{array}{c}\text { Dispersions } \\
\text { coëfficient } \\
\text { Dunkelblau } \\
\text { Roth } \\
\end{array}$ \\
\hline & & 74,88 & 98,28 & 125,75 & 148,50 & & & 2,5 \\
\hline ( & 4,84 & 74,47 & 97,14 & 124,87 & 147,18 & & & 2,46 \\
\hline & 6,37 & 73,91 & 97,02 , & 124,45 & 145,85 & & & 2,48 \\
\hline & 8,19 & 78,56 & 96,52 & 123,70 & 145,34 & & & 2,49 \\
\hline & 11,05 & 73,41 & 96,43 & 123,27 & 145,08 & & & $2,49^{\circ}$ \\
\hline
\end{tabular}

Tafel III.

Saccharin in Aethylalkohol.

\begin{tabular}{|c|c|c|c|c|c|c|c|}
\hline \multirow{2}{*}{$\begin{array}{c}\text { Nr. } \\
\text { der } \\
\text { Lö- } \\
\text { sung }\end{array}$} & \multirow{2}{*}{$\begin{array}{c}\text { In } 100 \\
\text { Gewichtsth. } \\
\text { Lösung } \\
\text { Gewichtsth. } \\
\text { Saccharin } \\
\text { p }\end{array}$} & \multirow{2}{*}{$\begin{array}{l}\mathrm{d}_{4}^{20} \text { der } \\
\text { Lösung }\end{array}$} & \multicolumn{5}{|c|}{$\alpha$ für $1=2 . \quad \mathrm{t}=20^{\circ}}$. \\
\hline & & & $\begin{array}{c}\text { Roth } \\
\lambda=665,5\end{array}$ & $\begin{array}{c}\mathrm{D} \\
\hat{i}=589,3\end{array}$ & $\begin{array}{c}\text { Grün } \\
\lambda=533,0\end{array}$ & $\begin{array}{c}\text { Hell- } \\
\text { blau } \\
\lambda=488,5\end{array}$ & $\begin{array}{c}\text { Dunkel- } \\
\text { blau } \\
\lambda=448,2\end{array}$ \\
\hline 1. & 1,55 & 0,7983 & 1,86 & 2,40 & 3,10 & 3,73 & $4, \overline{5} 5$ \\
\hline 2. & 1,97 & 0,8000 & 2,29 & 3,04 & 3,87 & 4,69 & 5,79 \\
\hline 3. & 2,48 & 0,8018 & 2,88 & 3,83 & 4,87 & 5,92 & 7,26 \\
\hline fa. & 3,00 & 0,8041 & 3,52 & 4,65 & 5,92 & 7,21 & 8,84 \\
\hline (b. & 3,02 & 0,8049 & 3,46 & $4,6 \mathfrak{j}$ & 5,92 & 7,17 & 8,82 \\
\hline 5. & 4,00 & 0,8079 & 4,65 & 6,20 & 7,88 & 9,58 & 11,78 \\
\hline
\end{tabular}




\begin{tabular}{|c|c|c|c|c|c|c|c|}
\hline $\mathrm{Nr}$. & \multirow[b]{2}{*}{$\mathrm{p}$} & & \multicolumn{2}{|l|}{ a] $20^{\circ}$} & \\
\hline $\begin{array}{l}\text { der } \\
\text { Lö- } \\
\text { sung }\end{array}$ & & Roth & D & Grün & Hellblau & $\begin{array}{c}\text { Dunkel- } \\
\text { blau }\end{array}$ & $\begin{array}{c}\text { Dispersions } \\
\text { coëffleient } \\
\text { Dunkelblau } \\
\text { Roth }\end{array}$ \\
\hline 1 & 155 & 7515 & 9697 & $19 \overline{9} 96$ & ל750 15 & 18385 & c) 45 \\
\hline 1. & 1,05 & $\begin{array}{l}70,15 \\
72,66\end{array}$ & $\begin{array}{l}96,98 \\
96,48\end{array}$ & 19267 & $\begin{array}{l}100,12 \\
148.90\end{array}$ & 183.71 & 2,53 \\
\hline 3. & 2,48 & 72,42 & 96,30 & 122,51 & 148,86 & 182,55 & 2,52 \\
\hline 4. & 3,01 & 72,11 & 96,08 & 122,32 & 148,56 & 182,40 & 2,53 \\
\hline כ. & 4,00 & 72,02 & 95,93 & 121,93 & 148,24 & 182,35 & 2,53 \\
\hline
\end{tabular}

Tafel IV.

Succharin in Aceton.

\begin{tabular}{|c|c|c|c|c|c|c|c|c|c|}
\hline \multirow{3}{*}{\multicolumn{3}{|c|}{$\begin{array}{cc} & \text { In } 100 \\
\text { Nr. } & \text { Gewichtsth. } \\
\text { der } & \text { Lösung } \\
\text { Lö- } & \text { Gewichtsth. } \\
\text { sung } & \text { Saccharin } \\
& p \\
=- & \text { p }\end{array}$}} & \multirow{3}{*}{$\begin{array}{l}\mathrm{d}_{4}^{20} \text { der } \\
\text { Lösung }\end{array}$} & \multicolumn{6}{|c|}{$\alpha$ für $1=2 . \quad t=20^{\circ}$. } \\
\hline & & & & Roth & $\mathrm{D}$ & Grün & & 11- & $\begin{array}{c}\text { Dunkel- } \\
\text { blau }\end{array}$ \\
\hline & & & & $\lambda=66 \check{0}, \bar{o}^{\prime}$ & $=589,3 \lambda=$ & $=\tilde{\mathbf{g}} 33, \mathrm{C}$ & $\lambda=4$ & & $\lambda=448,2$ \\
\hline 1. & 1,5 & 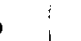 & 0,7980 & 2,03 & 2,63 & 3,25 & 3 & 91 & 5,00 \\
\hline 2. 1 & 1,6 & & 0,7982 & 2,17 & 2,79 & 3,57 & & 29 & 5,31 \\
\hline 3. & 1,7 & $\therefore$ & 0,7984 & 2,28 & 2,93 & 3,74 & & 50 & 5,59 \\
\hline 4. ! & 1,8 & ? & 0,7989 & 2,42 & 3,13 & 3,99 & & 80 & 5,97 \\
\hline 5. & 1,9 & : & 0,7991 & 2,47 & 3,20 & 4,08 & & 93 & 6,13 \\
\hline Nr. & & & & & {$[\alpha] 20^{\circ}$} & & & & \\
\hline $\begin{array}{l}\text { der } \\
\text { Lö- } \\
\text { sung }\end{array}$ & $p$ & Roth & $\mathrm{D}$ & Grün & Hellblau & $\underset{b l}{\text { Dun }}$ & & & $\begin{array}{l}\text { persions- } \\
\text { efflcient } \\
\text { nkelblau- } \\
\text { Rotb }\end{array}$ \\
\hline 1. & 1,505 & 82,06 & 106,31 & 135,82 & 163,31 & & & & 2,46 \\
\hline 2. & 1,66 & 81,71 & $105,2:$ & 134,72 & 161,80 & 200 & & & 2,45 \\
\hline 3. & 1,75 & 81,53 & 104.8 & 133,83 & 161,03 & 200 & & & 2,45 \\
\hline 4. & 1,87 & 80,85 & 104,6 & 133,61 & 160,75 & 195 & & & 2,47 \\
\hline 5. & 1,92 & 80,49 & 104,28 & 132,82 & 160,66 & 199 & & & 2,48 \\
\hline
\end{tabular}


324 Rimbach u. Heiten, Eigenschaften, Drehungsvermögen

Tafel V.

Saccharin in Eisessig.

\begin{tabular}{|c|c|c|c|c|c|c|c|}
\hline \multirow{2}{*}{\multicolumn{2}{|c|}{$\begin{array}{c:c} & \text { In } 100 \\
\text { Nr. } & \text { Gewichtsth. } \\
\text { der } & \text { Lösung } \\
\text { Lö- } & \text { Gewichtsth. } \\
\text { sung } & \text { Saccharin } \\
& p\end{array}$}} & \multirow{2}{*}{$\begin{array}{l}\mathrm{d}_{4}^{20} \text { der } \\
\text { Lösung }\end{array}$} & \multicolumn{5}{|c|}{$\alpha$ für $1=2 . \quad t=20^{\circ}$. } \\
\hline & & & $\begin{array}{c}\text { Roth } \\
\lambda=665,5\end{array}$ & $\begin{array}{c}\mathrm{D} \\
\lambda=589,3\end{array}$ & $\left\{\begin{array}{c}\text { Grün } \\
\lambda=533,0\end{array}\right.$ & $\begin{array}{c}\text { Hell- } \\
\text { blau } \\
\lambda=488,5\end{array}$ & $\begin{array}{c}\text { Dunkel- } \\
\text { blau } \\
\lambda=448,2\end{array}$ \\
\hline 1. & 1,33 & 1,0626 & 2,27 & 2,86 & 3,77 & 4,44 & 5,51 \\
\hline 2. & 1,39 & 1,0630 & 2,31 & 2,96 & 3,80 & 4,52 & 5,63 \\
\hline 3. & 1,45 & 1,0631 & 2,34 & 3,08 & 3,96 & 4,67 & 5,85 \\
\hline 4. ! & 1,59 & 1,0632 & $2, \overline{5} 4$ & 3,37 & 4,33 & 5,10 & 6,32 \\
\hline 5. & 1,66 & 1,0633 & 2,63 & 3,49 & 4,48 & 5,33 & 6,56 \\
\hline
\end{tabular}

\begin{tabular}{|c|c|c|c|c|c|c|c|}
\hline \multirow{2}{*}{$\begin{array}{c}\text { Nr. } \\
\text { der } \\
\text { Lö- } \\
\text { sung }\end{array}$} & \multirow[b]{2}{*}{$p$} & \multicolumn{6}{|c|}{$\lfloor\alpha] 20^{\circ}$} \\
\hline & & Roth & $\mathrm{D}$ & Griin & Hellblau & $\begin{array}{c}\text { Dunkel- } \\
\text { blau }\end{array}$ & $\begin{array}{c}\text { Dispersions- } \\
\text { coëfficient } \\
\text { Dunkelblau- } \\
\text { Roth }\end{array}$ \\
\hline 1. & 1,33 & 80,31 & 101,18 & 133,38 & 157,08 & 194,94 & 2,43 \\
\hline 2. & 1,39 & 78,17 & 100,17 & 128,59 & 152,96 & 190,53 & 2,44 \\
\hline 3. & 1,45 & 75,90 & 99,88 & 128,44 & 151,50 & 189,70 & 2,50 \\
\hline 4. & 1,59 & 75,20 & 99,57 & 128,08 & 150,93 & 187,05 & 2,49 \\
\hline 5. i & 1,66 & 74,47 & 98,78 & 126,63 & 150,66 & 185,71 & 2,49 \\
\hline
\end{tabular}

In sämmtlichen angewendeten Lösungsmitteln ist die Drehung höher als in Wasser; ferner erfolgt gleichmässig in allen Lösungsmitteln, mit steigender Concentration eine stetige Abnahme der Drehung. Letzeres allerdings in sehr verschiedenem Grade; je mehr ein Lösungsmittel, bei für alle Lösungsmittel gleicher, kleiner Anfangsconcentration, die Drehung uberhaupt hinaufsetzt, desto rascher findet in ihm, bei zunehmender Concentration, auch der Drehungsabfall statt. Nachstehende kleine graphische Darstellung, mit den Procentgehalten der Lösungen in der Abscissenaxe, den specifischen Drehungen als Ordinaten, bringt dies übersichtlich zum Ausdruck.

Die Rotationsdispersionen sind iberall normal und die Dispersionscoëfficienten fur die verschiedenen Concentrationen von ziemlicber Constanz. 
Eine weitere Verfolgung der Drehungscurven bez. eine Ausdehnung der Versuche auf andere Lösungsmittel war bei der ziemlich begrenzten Löslichkeit des Saccharins leider nicht thunlich.

Die Drehung des Saccharins, wie sie in obiger Tafel angefuhrt ist, kann eigentlich, wenigstens in wässrigen Lösungen, nicht als constant angesehen werden; denn das Saccharin geht

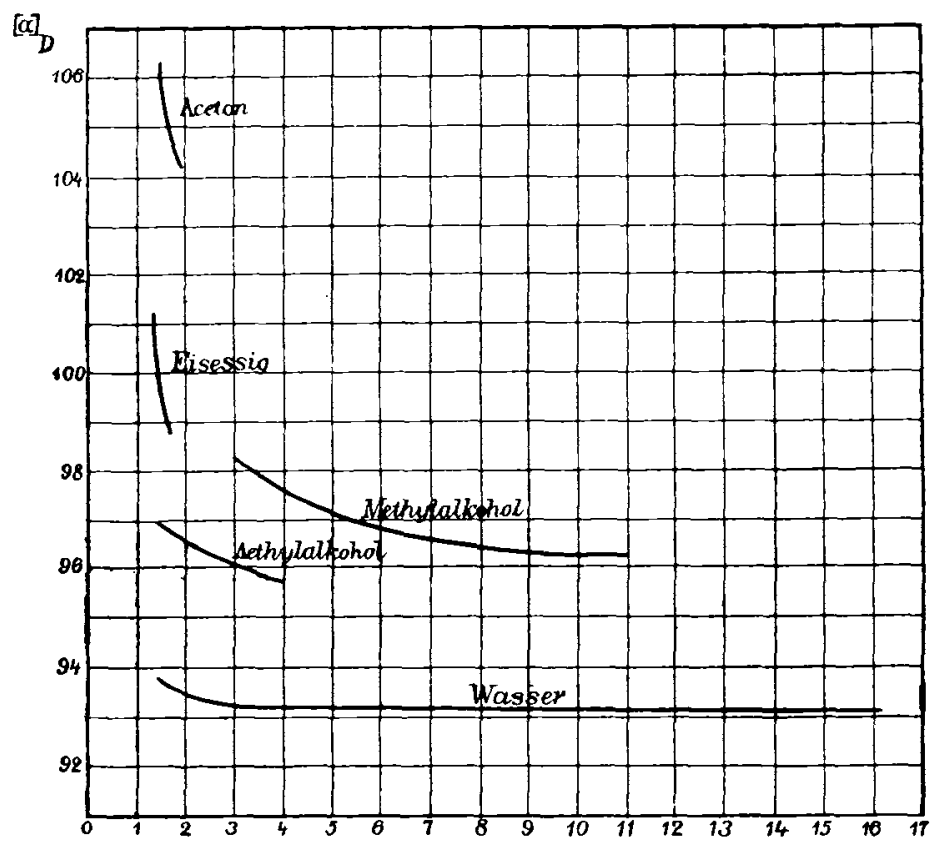

in wässrigen Lösungen zum Theil in Saccharinsäure uber, und da diese linksdrehend ist, so muss die Rechtsdrehung des Saccharins heruntergedrückt werden. Diese Thatsache ist bekannt, bereits untersucht aber von nur geringem Einflusse auf die Drehung gefunden worden. Es fanden Schnelle und Tollens ${ }^{8}$ ) fur eine zehnprocentige wässrige Saccharinlösung:

8) Schnelle und Tollens, diese Annalen $270,85$. 
326 Rimbach u. Heiten, Eigenschaften, Drehungsvermögen

$$
\begin{array}{lllll}
\text { Nach } 8 \text { Minuten } & 3 \text { Tagen } & 4 \text { Tagen } & 7 \text { Tagen } & 11 \text { Tagen } \\
{[\alpha]_{D}+94,2} & +90,7 & +90,5 & +88,9 & +88,7
\end{array}
$$

Wir haben diese. Versuche, unter Verwendung kürzerer Anfingsintervalle, wiederbolt und fanden bei einer Lösung, wo $\mathrm{C}=4,0$ war,

$$
\begin{array}{ccccccc}
\text { nach } 20 \text { Minuten } & \mathbf{3} & \mathbf{1 6} & 66 & 92 & 97 & 112 \text { Stunden } \\
{[\alpha]_{\mathrm{D}}+93,97} & 93,75 & 93,75 & 91,00 & 89,38 & 89,00 & 88,75 \\
& \multicolumn{5}{c}{\text { bei }} & 22^{\circ} .
\end{array}
$$

Temperaturerböhung beschleunigte die Reaction nicht merklich. Erbielt man die Lösung mit der Drehung $+88,75$ constant auf $35^{\circ}$, so war die Drehung

$$
\begin{array}{cccc}
\text { nach } 2 \text { Stunden } & 5 \text { Stunden } & 8 \text { Stunden } \\
{[\alpha]_{D}} & 88,62 & 88,37 & 88,37 .
\end{array}
$$

Mit diesen Befunden stehen die Ergebnisse der Leitfähigkeitsmessung im Einklange. Eine $1 / 8 n$-Saccharinlösung gab bei $25^{\circ}$

$\begin{array}{ccccc}\text { Nach } 1 / 3 \text { Stunde } & : / 4 \text { Stunde } & 1 / 2 \text { Stunden } & 2 \text { Stunden } \\ 1000 \times 0,0625 & 0,0637 & 0,0678 & 0,0701 \\ \text { nach } 4 \text { Stunden } & 26 \text { Stunden } & 76 \text { Stunden } & 172 \text { Stunden } \\ 1000 \times 0,0793 & 0,1443 & 0,1912 & 0,2282 .\end{array}$

Das Aequivalentleitvermögen $A$ steigt während dieser Zeit demnach nur von 0,50 bis 1,83 an.

Die Verschiebung des Gleichgewichtes

$$
\mathrm{C}_{6} \mathrm{H}_{19} \mathrm{O}_{6} \longleftrightarrow \mathrm{C}_{6} \mathrm{H}_{10} \mathrm{O}_{3}+\mathrm{H}_{8} \mathrm{O}
$$

von rechts nach links erfolgt also so langsam, dass sie für die Drehungsbestimmungen des Saccharins praktisch nicht ins Gewicht fällt.

\section{Saccharinsaure Salze.}

Von saccharinsauren Salzen stellten wir neben dem bereits von Kiliani ${ }^{9}$ ) und Scheibler ${ }^{10}$ ) beschriebenen Kalium- und

9) Kiliani, Ber. d. deutsch. chem. Ges. 15, 2955.

10) Scheibler, Ber. d. deutsch. chem. Ges. 13, 2215. 
Natriumsaccharinat das noch nicht bekannte Rubidiumsaccharinat her. $Z$ u einer genau abgewogenen Menge Saccharin wurde die äquivalente Menge titrirte Natrium-, Kalium- bezw. Rubidiumcarbonatlösung binzugegeben. Aus der concentrirten Lösung schieden sich das Kalium- und Rubidiumsalz in schön ausgebildeten Krystallen ab. Das schwer krystallisirende Natriumsalz wurde aus der concentrirten Lösung mit Alkohol gefällt; nach öfterem Umrübren setzte sich eine weisse, fadenziehende Masse ab, die bei $100^{\circ}$ getrocknet ein weisses Pulver bildete.

Die Analyse der drei Salze ergab ihre Reinheit.

$\begin{array}{cc}\text { Berechnet für } & \text { Gefunden } \\ \mathrm{C}_{6} \mathrm{H}_{11} \mathrm{O}_{6} \mathrm{~K} & \\ 19,46 & 19,11 \\ \mathrm{C}_{0} \mathrm{H}_{11} \mathrm{O}_{6} \mathrm{Na} & \\ 12,42 & 12,21 \\ \mathrm{C}_{6} \mathrm{H}_{11} \mathrm{O}_{6} \mathrm{Rb} & \\ 32,30 & 32,16\end{array}$

Die Alkalien sind hierbei als Sulfate gewogen worden, nachdem der organische Theil der Substanz durch gelindes Gluhen, Auslaugen des kohligen Ruckstandes mit Wasser und nochmaliges Glühen desselben zerstört war.

Die von Herrn Dr. Fock bestimmten krystallographischen Constanten des sehr schön krystallisirenden Kalium- und Rubidiumsalzes finden sich im Folgenden.

\section{Kaliumsaccharinat.}

Krystallsystem: Monoklin.

$$
\begin{aligned}
a: b: c & =1,2658: 1: 0,8306 . \\
\beta & =9^{\circ} 44^{\circ} .
\end{aligned}
$$

Beobachtete Formen:

$$
\mathrm{a}=(100), \mathrm{c}=(001), \mathrm{p}=(111), 0=(\overline{1} 11) \text {. }
$$

Die Krystalle sind meist dicktafelförmig nach $c(001)$ und bis zu $10 \mathrm{~mm}$ lang bezw. breit und $5 \mathrm{~mm}$ dick. Von den angegebenen Formen treten $o$ (i11) und a (100) in der Regel 
gegen p (111) zuräck; a (100) fehlt auch vielfach ganz, jedoch war ein einzelner grosser Krystall tafelförmig nach dieser Fläche ausgebildet.

$$
\begin{aligned}
& \text { Beobachtet: } \\
& \mathrm{a}: \mathrm{c}=(100):(001)=85^{\circ} 16^{\prime} \\
& 0: 0=(\overline{1} 11):(\overline{1} 1)=95^{\circ} 18^{\circ} \\
& \mathrm{a}: 0=(\overline{100}):(\overline{1} 11)=56^{\circ} 21^{\circ} \\
& \mathrm{c}: \mathrm{o}=(001):(\overline{1} 11)=70^{\circ} 21^{\circ} \\
& \mathrm{p}: \mathrm{p}=(111):(1 \overline{1} 1)=91^{\circ} 8^{\circ} \\
& \mathbf{c}: \mathbf{p}=(001):(111)=65^{\circ} 21^{\circ} \\
& \mathrm{a}: \mathrm{p}=(100):(111)=53^{\circ} 35^{\prime} \\
& 0: p=(111):(111)=70^{0} 4^{\prime} \\
& 0: \mathrm{p}=(11 \overline{1}):(111)=44^{\circ} 18^{\prime} \\
& \text { - } \\
& 70^{\circ} 22^{\prime} \\
& 90^{\circ} 58^{\prime} \\
& 65^{\circ} 19^{\prime} \\
& 53^{\circ} 26^{\prime} \\
& 70^{\circ} 11^{4} \\
& 44^{\circ} 19^{\prime}
\end{aligned}
$$

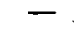

Spaltbarkeit vollkommen nach a (100), deutlich nach c (001). Ebene der optischen Axen = Symmetrieebene (010). Durch c (001) tritt eine Axe scheinbar circa $24^{\circ}$ gegen die Normale geneigt im spitzen Winkel $\beta$ aus. Durch a (100) tritt die andere Axe scheinbar circa $30^{\circ}$ gegen die Normale geneigt im stumpfen Winkel $\beta$ aus.

Rubidiumsaccharinat (isomorph mit dem Kaliumsalz).

Krystallsystem: Monoklin.

$$
\begin{aligned}
\mathrm{a}: \mathrm{b}: \mathrm{c} & =1,2297: 1: 0,8171 . \\
\beta & =92^{0} 16^{\prime} .
\end{aligned}
$$

Beobachtete Formen:

$$
\mathrm{c}=(001), \mathrm{p}=(111), 0=(\overline{1} 11) \text { und } \mathrm{a}=(100) \text {. }
$$

Die Krystalle sind zum grössten Theile tafelförmig nach c (001), zum Theil auch von verzerrter Ausbildung und im Durchschnitte bis zu $4 \mathrm{~mm}$ lang bezw. breit und $2 \mathrm{~mm}$ dick. Ein grösseres verzerrtes Individuum zeigte Dimensionen von $1 \mathrm{~cm}$. Von den Randflächen herrscht $p$ (111) regelmässig vor, wăhrend o (111) und a (100) nur ganz untergeordnet erscheinen und vielfach auch ganz fehlen. 


$\begin{array}{lcc} & \text { Beobachtet: } & \text { Berechnet: } \\ \mathrm{c}: \mathrm{p}=(001):(11)=66^{\circ} 2^{\prime} & - \\ \mathrm{c}: \mathrm{o}=(001):(\overline{1} 11)=68^{\circ} 28^{\prime} & - \\ \mathrm{p}: \mathrm{p}=(11):(1 \overline{1} 1)=90^{\circ} 18^{\prime} & - \\ \mathrm{a}: \mathrm{c}=(100):(001)=87^{\circ} 30^{\prime} & 87^{\circ} 44^{\prime} \\ \mathrm{a}: \mathrm{p}=(100):(111)=53^{\circ} 50^{\prime} & 53^{\circ} 41^{\prime} \\ \mathrm{a}: 0=(\overline{100}):(\overline{1} 11)=55^{\circ} 37^{\prime} & 55^{\circ} 7^{\prime} \\ 0: 0=(\overline{1} 1):(\overline{1} 11)=92^{\circ} \mathrm{ca} . & 92^{\circ} 23^{1 / 2} \mathrm{2}^{\prime} \\ 0: \mathrm{p}=(\overline{1} 11):(111)=71^{\circ} \mathrm{ca} . & 71^{\circ} 12^{\prime} .\end{array}$

Spaltbarkeit vollkommen nach c (001).

Ebene der optischen Axen=Symmetrieebene (010); durch c (001) tritt eine Acbse scheinbar circa $13^{\circ}$ gegen die Normale geneigt im spitzen Winkel $\beta$ aus.

Die Drehungsmessungen der wässrigen Lösungen der drei Salze ergaben folgende Werthe:

\section{Natriumsaccharinat.}

\begin{tabular}{|c|c|c|c|c|c|c|}
\hline \multirow{2}{*}{$\begin{array}{l}\text { Nr. } \\
\text { der } \\
\text { Lö- } \\
\text { sung }\end{array}$} & \multirow{2}{*}{$\begin{array}{c}\text { g Salz in } \\
100 \mathrm{ccm} \\
\text { Lösung } \\
\text { c }\end{array}$} & \multicolumn{4}{|c|}{$\alpha$ für $1=-2 ; t=20^{\circ}$} & \multirow[b]{2}{*}{$\begin{array}{l}\text { Dunkel } \\
\text { blau }\end{array}$} \\
\hline & & Roth & D & Grïn & Hellblan & \\
\hline 1. & 5,34 & $\quad-0,43$ & $-0,61$ & $-0,76$ & $-0,92$ & $-1,23$ \\
\hline 2. & 8,90 & $-0,76$ & $-1,02$ & $-1,39$ & $-1,66$ & $-2,11$ \\
\hline 3. & 16,00 & $-1,38$ & $-1,85$ & $-2,55$ & $-3,06$ & $-3,86$ \\
\hline 4. & 22,40 & $-2,00$ & $-2,67$ & $-3,76$ & $-4,35$ & $-5,47$ \\
\hline 5̆. & 28,00 & $-2,59$ & $-3,47$ & $-4,82$ & $-5,49$ & $-6,97$ \\
\hline
\end{tabular}

\begin{tabular}{|c|c|c|c|c|c|c|c|}
\hline Nr. & $g \mathrm{Salz}$ in & \multicolumn{6}{|c|}{$[\alpha] 20^{\circ}$} \\
\hline $\begin{array}{l}\text { Lö- } \\
\text { sung }\end{array}$ & $\begin{array}{c}\text { Lösung } \\
\text { c }\end{array}$ & Roth & D & Grün & $\begin{array}{l}\text { Hell- } \\
\text { blau }\end{array}$ & $\begin{array}{c}\text { Dunkel- } \\
\text { blau }\end{array}$ & $\begin{array}{c}\text { Dispersions } \\
\text { coefticient } \\
\text { Dunkelban- } \\
\text { Rotb }\end{array}$ \\
\hline 1. & 5,34 & $-4,05$ & $-5,71$ & $-7,12$ & $-8,62$ & $-11,52$ & 2,84 \\
\hline 2. & 8,90 & $-4,25$ & $-5,73$ & $-7,81$ & $-9,33$ & $-11,86$ & 2,79 \\
\hline 3. & 16,00 & $-4,31$ & $-5,78$ & $-7,97$ & $-9,56$ & $-12,06$ & 2,80 \\
\hline 4. & 22,40 & $-4,47$ & $\sim \overline{\boldsymbol{5}}, 95$ & $-8,40$ & $--9,71$ & $-12,20$ & 2,74 \\
\hline 5. & 28,00 & $-4,62$ & $-6,20$ & $-8,61$ & $-9,82$ & $-12,45$ & 2,76 \\
\hline
\end{tabular}

Auvalen der Chemie \$50. Bd. 
Kaliumsaccharinat.

\begin{tabular}{|c|c|c|c|c|c|c|}
\hline $\mathrm{Nr}$. & $\mathrm{g}$ Salz in & \multicolumn{5}{|c|}{$\alpha$ für $1=2 ; t=20^{\circ}$} \\
\hline $\begin{array}{l}\text { I,ö- } \\
\text { sung }\end{array}$ & $\begin{array}{c}\text { Lösung } \\
\mathrm{c} \\
\end{array}$ & Roth & D & Grün & Hellblau & $\begin{array}{c}\text { Duukel- } \\
\text { blau } \\
\end{array}$ \\
\hline 1. & 10,02 & $-0,72$ & $-1,11$ & $-1,45$ & $-1,80$ & $-2,46$ \\
\hline 2. & 11,96 & $-0,97$ & $-1,37$ & $-1,79$ & $-2,21$ & $-2,97$ \\
\hline 3. & 16,00 & $-1,35$ & $-1,84$ & $-2,40$ & $-2,97$ & $-4,06$ \\
\hline 4. & 20,00 & $-1,75$ & $-2,44$ & $-3,27$ & $-3,99$ & $-5,14$ \\
\hline$\check{\text { o. }}$ & 23,00 & $-2,08$ & $-2,87$ & $-3,88$ & $-4,67$ & $-6,09$ \\
\hline 6. & $2 \overline{5}, 0 \overline{0}$ & $-2,29$ & $-3,20$ & $-4,28$ & $-5,12$ & $-6,75$ \\
\hline
\end{tabular}

\begin{tabular}{|c|c|c|c|c|c|c|c|}
\hline Nr. & $\mathrm{g} \mathrm{Salz}$ in & \multicolumn{6}{|c|}{$[\alpha] 20^{\circ}$} \\
\hline $\begin{array}{l}\text { der } \\
\text { Lö- } \\
\text { sung }\end{array}$ & $\begin{array}{c}100 \mathrm{ccm} \\
\text { Lösung } \\
\mathrm{c}\end{array}$ & Roth & D & Grün & $\begin{array}{l}\text { Hell- } \\
\text { blau }\end{array}$ & $\begin{array}{c}\text { Dunkel- } \\
\text { blau }\end{array}$ & $\begin{array}{c}\text { Dispersions- } \\
\text { coefficient } \\
\text { Dunkelblau- } \\
\text { Roth }\end{array}$ \\
\hline 1. & 10,02 & $-3,59$ & $-5,54$ & $-7,23$ & $-8,98$ & $-12,27$ & 3,42 \\
\hline 2. & 11,96 & $-4,05$ & $-5,73$ & $-7,48$ & $-9,2$ & $-12,42$ & 3,07 \\
\hline 3. & 16,00 & $-4,23$ & $-5,75$ & $-7,50$ & $-9,2$ & $-12,68$ & 3,00 \\
\hline 4. & 20,00 & $-4,37$ & $-6,10$ & $-8,17$ & $-9,9$ & $-12,85$ & 2,94 \\
\hline ঠ. & 23,00 & $--4,52$ & $-6,25$ & $-8,43$ & $-10,1$ & $-13,24$ & 2,93 \\
\hline 6. & 25,05 & $-4,57$ & $-6,39$ & $-8,51$ & $-10,2$ & $-13,47$ & $2,9 j$ \\
\hline
\end{tabular}

Rubidiumsaccharinat.

\begin{tabular}{|c|c|c|c|c|c|c|}
\hline \multirow{2}{*}{$\begin{array}{l}\text { Nr. } \\
\text { der } \\
\text { Lö- } \\
\text { sung }\end{array}$} & \multirow{2}{*}{$\begin{array}{c}\text { g Salz in } \\
100 \mathrm{ccm} \\
\text { Lösung } \\
\text { c }\end{array}$} & \multicolumn{5}{|c|}{$\alpha$ für $1=2 ; t=20^{\circ}$} \\
\hline & & Roth & $\mathrm{D}$ & Grïn & Hellblau & $\begin{array}{c}\text { Dunkel- } \\
\text { blau }\end{array}$ \\
\hline 1. & 8,00 & $-0,32$ & $-0,59$ & $-0,69$ & $-0,89$ & $-1,19$ \\
\hline 2. & 12,00 & $-0,545$ & $-0,95$ & $-1,13$ & $-1,46$ & $-1,99$ \\
\hline 3. & 16,00 & $-0,81$ & $-1,36$ & $-1,72$ & $-2,17$ & $-2,92$ \\
\hline $4-$ & 20,00 & $-1,13$ & $-1,86$ & $-2,46$ & $-3,17$ & $-4,12$ \\
\hline 5. & 24,00 & $--1,72$ & $-2,52$ & $-3,27$ & $-4,03$ & $-5,34$ \\
\hline
\end{tabular}




\begin{tabular}{|c|c|c|c|c|c|c|c|}
\hline \multirow{2}{*}{$\begin{array}{l}\text { Nr. } \\
\text { der } \\
\text { Lö- } \\
\text { sung }\end{array}$} & \multirow{2}{*}{$\begin{array}{c}g \text { Salz in } \\
100 \mathrm{ccm} \\
\text { Lösung } \\
\mathrm{c}\end{array}$} & \multicolumn{6}{|c|}{$[\alpha] 20^{\circ}$} \\
\hline & & Roth & D & Grün & $\begin{array}{l}\text { Hell- } \\
\text { blau }\end{array}$ & $\begin{array}{c}\text { Dunkel- } \\
\text { blau }\end{array}$ & $\begin{array}{l}\text { Dispersious } \\
\text { coéfticient } \\
\text { Dunkelblau. } \\
\text { Roth }\end{array}$ \\
\hline 1 & 8.00 & $\begin{array}{l}= \\
-2,02\end{array}$ & -371 & & $=\therefore-$ & -745 & -369 \\
\hline 2. & $\begin{array}{r}0,00 \\
12,00\end{array}$ & $-2,27$ & $-3,96$ & $\begin{array}{r}4,04 \\
-4,71\end{array}$ & $\begin{array}{r}-6,00 \\
-6,07\end{array}$ & $-8,28$ & $\begin{array}{l}5,00 \\
3,65\end{array}$ \\
\hline 3. & 16,00 & $-2,53$ & $-4,25$ & $-5,37$ & $-6,78$ & $-9,12$ & 3,60 \\
\hline 4. & 20,00 & $-2,82$ & $-4,65$ & $-6,15$ & $-7,92$ & $-10,30$ & 3,65 \\
\hline 5. & 24,00 & $-3,58$ & $--5,25$ & $-6,82$ & $-8,40$ & $-11,13$ & 3,10 \\
\hline
\end{tabular}

Bei allen drei Salzen wächst daher mit steigender Concentration die Linksdrehung, während in den Lösungen des freien Saccharins unter gleichen Umständen die Rechtsdrehung abnimmt. Die oben gefundene specifische Drehung für Natriumsaccharinat weicht nun von den Angaben Scheibler's ${ }^{11}$ ), die er selbst allerdings als nur vorläufige bezeichnet, vollständig ab. Nach Scheibler gilt für Natriumsaccharinat $[\alpha]_{\mathrm{D}}=-17,2$, während wir fur die Concentration 5 bis -28 $[\alpha]_{\mathrm{D}} \mathrm{zu}-5,71$ bis $-6,20$ fanden.

Die Molekulardrehungen [M] der drei Salze sind, gemessen fur die D-Linie, folgende, wobei in üblicher Weise, wenn $P$ das Molekulargewicht ist,

$$
[\mathrm{M}]=[\alpha] \cdot P \cdot 10^{-2}
$$

gesetzt ist.

Natriumsaccharinat.

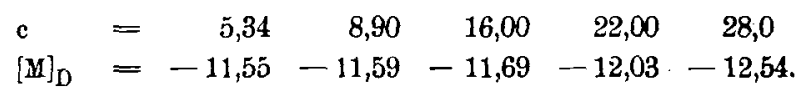

Kaliumsaecharinat.

$$
\begin{aligned}
& \text { c }=\begin{array}{rrrrrr}
10,02 & 11,96 & 16,00 & 20,00 & 23,00 & 25,05
\end{array} \\
& {[M]_{D}=-12,09-12,51 \quad-12,55-13,32-13,64-13,95 \text {. }}
\end{aligned}
$$

Rubidiumsaccharinat.

$$
\begin{array}{lrrrrrr}
\mathrm{c} & = & 8,00 & 12,00 & 16,00 & 20,00 & 24,00 \\
{[\mathbf{M}]_{\mathrm{D}}} & = & -9,82 & -10,48 & -11,24 & -12,31 & -13,89 .
\end{array}
$$

1) Scheibler, Ber. d. deutsch. chem. Ges. 13, 2212. 
Die nahe Uebereinstimmung der Molekulardrehungen der drei in correspondirenden Lösungen wohl ziemlich gleichstark dissociirten Salze entspricht dem Landolt-Oudemans'schen Satze ${ }^{12}$ ) und beweist zugleich schon, dass bei unserer Messung des Natriumsalzes ein Irrthum nicht vorliegen kann. Zur völligen Sicherheit wurde jedoch das Natriumsaccharinat ganz von Neuem nach der Eingangs beschriebenen Methode dargestellt. Die Analyse des neuen Präparates ergab vollständige Reinheit und für eine Lösung von $8,8952 \mathrm{~g}$ in $100 \mathrm{ccm}$ Wasser fand sich die specifische Drehung genau wiederum: $[\alpha]_{\mathfrak{D}}^{20}=-5,73$ wie bei den früheren Versuchen. Es muss also entweder ein Irrthum Scheibler's vorliegen, oder eine Unreinheit seiner Substanz.

Den Einfluss der Temperatur endlich auf die Drehung der Saccharinate in wässriger Lösung haben wir beim Kalium- und Natriumsalz untersucht, und zwar so, dass eine bei $20^{\circ}$ hergestellte Lösung bestimmter Concentration in der Röhre auf eine höbere Temperatur gebracht und bei dieser direct gemessen wurde. Natürlich ändert sich durch die Ausdehnung die Concentration. Die in der zweiten Spaite gegebenen Werthe fur $c$ sind rechnerisch aus der Anfangsconcentration und zwar so ermittelt, dass für die betreffenden Salzlösungen als Ausdehnungscoëfficient für das Intervall $20-35^{\circ} \mathrm{der}$ Werth 0,0005 ; $35-50^{\circ} 0,0006 ; 50-60^{\circ} 0,0007$ eingesetzt wurde, Mittelwerthe aus den bekannten Ausdehnungscoëfficienten für Robrzucker- bezw. Natriumchloridlösungen entsprechender Concentration. Die in der Wahl dieser Mittelwerthe liegende Unsicherheit beeinflusst im äussersten Falle (bei $60^{\circ}$ ) die Hundertstel der specifischen.Drehuug, liegt also völlig innerhalb der sonstigen Fehlergrenzen.

12) La nd olt, Ber. d. deutsch. chem. Ges. 6, 1077 (1873); Oudemans, diese Annalen 182, 33, 58 (1876); 197, 48, 66 (1879); Haedrich, Zeitschr. f. physik. Chem. 12, 476 (1893). 


\section{Drehung des Kaliumsaccharinats}

bei verschiedenen Temperaturen.

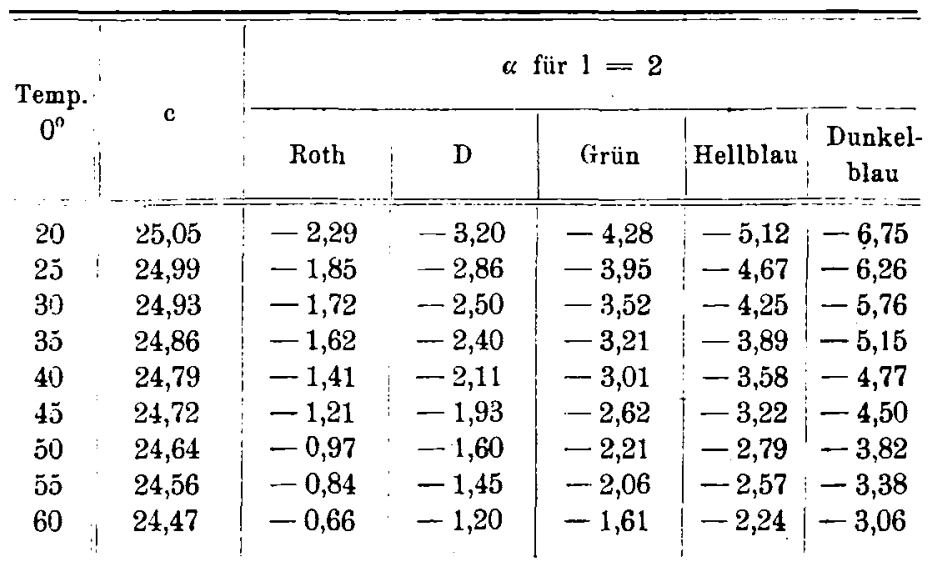

$[a]$

\begin{tabular}{|c|c|c|c|c|c|c|}
\hline $0^{\prime \prime}$ & Roth & D & Grün & $\begin{array}{l}\text { Hell- } \\
\text { blau }\end{array}$ & $\begin{array}{c}\text { Dunkel- } \\
\text { blaus }\end{array}$ & $\begin{array}{c}\text { Dispersions- } \\
\text { coetecient } \\
\text { Dunkelbau- } \\
\text { Roth }\end{array}$ \\
\hline 20 & $-4,57$ & $-6,39$ & $-8,54$ & $-10,22$ & $-13,47$ & 2,95 \\
\hline 25 & $-3,70$ & $-5,72$ & $-7,90$ & $-9,35$ & $-12,52$ & 3,38 \\
\hline 30 & $-3,4 \bar{j}$ & $-5,02$ & $-7,06$ & $-8,53$ & $-11,56$ & 3,35 \\
\hline 35 & $-3,26$ & $-4,83$ & $-6,46$ & $-7,82$ & $-10,36$ & 3,18 \\
\hline 40 & $-2,84$ & $-4,26$ & $-6,07$ & $-7,22$ & $-9,62$ & 3,39 \\
\hline 45 & $-2,45$ & $-3,90$ & $-5,30$ & $-6,51$ & $-9,10$ & - \\
\hline 50 & $-1,97$ & $-3,25$ & $-4,48$ & $-5,66$ & $-7,75$ & - \\
\hline 55 & $-1,71$ & $-2,95$ & $-4,19$ & $-5,23$ & $-6,88$ & - \\
\hline 60 & $-1,35$ & $-2,40$ & $-3,29$ & $-4,58$ & $-6,25$ & - \\
\hline
\end{tabular}


334 Rimbach u. Heiten, Eigenschaften, Drehungsvermögen

\section{Drehung des Natriumsaccharinats bei verschiedenen Temperaturen.}

\begin{tabular}{|c|c|c|c|c|c|c|}
\hline \multirow{2}{*}{$\begin{array}{c}\text { Temp. } \\
0^{\circ}\end{array}$} & \multirow{2}{*}{ c } & \multicolumn{5}{|c|}{$\alpha$ für $1=2$} \\
\hline & & Roth & D & Grün & Hellblau & $\begin{array}{c}\text { Dunkel- } \\
\text { blau }\end{array}$ \\
\hline 20 & 24,33 & $-2,21$ & $-2,90$ & $-4,10$ & $-4,93$ & $-6,17$ \\
\hline 25 & 24,27 & $-1,92$ & $-2,75$ & $-3,63$ & $-4,65$ & $-\tilde{\mathbf{b}}, 6 \overline{\mathbf{b}}$ \\
\hline 30 & 24,21 & $-1,68$ & $-2,44$ & $-3,43$ & $-4,36$ & $-5,17$ \\
\hline 40 & 24,03 & $-1,41$ & $-1,97$ & $-2,86$ & $-3,65$ & $-4,72$ \\
\hline 50 & 23,93 & $-1,11$ & $-1,39$ & $-2,33$ & $-2,91$ & $-4,27$ \\
\hline 60 & 23,77 & $-0,83$ & $-1,25$ & $-1,94$ & $-2,39$ & $-3,61$ \\
\hline
\end{tabular}

\begin{tabular}{|c|c|c|c|c|c|c|}
\hline \multirow{2}{*}{$\begin{array}{c}\text { Temp. } \\
0^{0}\end{array}$} & \multicolumn{6}{|c|}{$[\alpha]$} \\
\hline & Roth & $\mathrm{D}$ & Grün & $\begin{array}{l}\text { Hell- } \\
\text { blau }\end{array}$ & $\begin{array}{c}\text { Dunkel- } \\
\text { blau }\end{array}$ & $\begin{array}{c}\text { Dispersions } \\
\text { coeffacient } \\
\text { Dunk+lblau } \\
\text { Roth }\end{array}$ \\
\hline 20 & $-4, \tilde{0} 4$ & $-5,96$ & $-8,43$ & $-10,13$ & $-12,68$ & 2,79 \\
\hline 25 & $-3,96$ & $-5,67$ & $-7,48$ & $-9,58$ & $-11,64$ & 2,94 \\
\hline 30 & $-3,47$ & $-5,04$ & $-7,08$ & $-9,01$ & $-10,68$ & 3,08 \\
\hline 40 & $-2,93$ & $-4,09$ & $-5,94$ & $-7,58$ & $-9,80$ & 3,34 \\
\hline 50 & $-2,32$ & $-2,90$ & $-4,87$ & $-6,08$ & $-8,92$ & - \\
\hline 60 & $-1,75$ & $-2,63$ & $-4,08$ & $-5,03$ & $-7,59$ & - \\
\hline
\end{tabular}

Mit wachsender Temperatur finden wir sowohl bei Kaliumwie bei Natriumsaccharinat eine beträchtliche Abnahme der specifischen Drehung bei allen Lichtarten.

Angeschlossen finden sich noch einige Beobachtungen tiber die Leitfähigkeit der Saccharinate.

Kaliumsacchararinat. $\mathrm{t}=25^{\circ}$.

$\begin{array}{rlcccc}v & = & 8 & 16 & 32 & 64 \\ 1000 x & = & 8,210 & 4,512 & 2,465 & 1,287 \\ A & = & 65,68 & 72,20 & 78,87 & 82,35 \\ \nabla & = & 128 & 256 & 512 & 1024 \\ 1000 x & = & 0,6644 & 0,3423 & 0,1783 & 0,09687 \\ A & = & 85,04 & 87,63 & 91,31 & 99,19 .\end{array}$


des Saccharins und der Alkalisaccharinate.

Rubidiumsaccharinat. $\mathrm{t}=25^{\circ}$.

$\begin{array}{rccccc}\mathrm{v} & = & 8 & 16 & 32 & 64 \\ 1000 x & = & 8,1514 & 5,3707 & 2,8352 & 1,4715 \\ \boldsymbol{A} & = & 65,21 & 85,93 & 90,73 & 94,18 \\ \mathrm{v} & = & 128 & 256 & 512 & 1024 \\ 1000 x & = & 0,75605 & 0,38502 & 0,1975 & 0,10184 \\ \boldsymbol{A} & = & 96,77 & 98,56 & 101,12 & 104,29 .\end{array}$

Auf Grund dieser Zablen ergiebt sich nach der Ostwaldschen Valenzregel die Basicităt der Saccharinsäure zu

$$
\left(A_{1024}-A_{3 \mathrm{~g}}\right) \cdot 10^{-1}=\left\{\begin{array}{l}
2,03 \text { (aus dem Kaliumsalz) } \\
1,36 \text { (aus dem Rubidiumsalz). }
\end{array}\right.
$$

Da die einbasische Natur diesẹs Körpers wohl zweifellos ist, so stellt sich hiernach die Saccharinsäure als eine recht schwache Säure dar, deren Alkalisalze bereits einer starken Hydrolyse unterliegen, das Rubidiumsalz weniger als die Kaliumverbindung. 